\title{
Social Network Analytics for Churn Prediction in Telco: Model Building, Evaluation and Network Architecture
}

\author{
María Óskarsdóttira, ${ }^{\mathrm{a}, *}$ Cristián Bravo ${ }^{\mathrm{b}}$, Wouter Verbeke ${ }^{\mathrm{c}}$, Carlos Sarraute ${ }^{\mathrm{d}}$, Bart Baesens ${ }^{\mathrm{a}, \mathrm{b}}$, Jan \\ Vanthienen $^{\mathrm{a}}$ \\ ${ }^{a}$ Dept. of Decision Sciences and Information Management, KU Leuven, Naamsestraat 69, 3000 Leuven, Belgium. \\ ${ }^{b}$ Dept. of Decision Analytics and Risk, University of Southampton, United Kingdom. \\ ${ }^{c}$ Faculty of Economic and Social Sciences and Solvay Business School, Vrije Universiteit Brussel, Belgium. \\ ${ }^{d}$ Grandata Labs, Bartolome Cruz, 1818 V. Lopez, Buenos Aires, Argentina.
}

\begin{abstract}
Social network analytics methods are being used in the telecommunication industry to predict customer churn with great success. In particular it has been shown that relational learners adapted to this specific problem enhance the performance of predictive models. In the current study we benchmark different strategies for constructing a relational learner by applying them to a total of eight distinct call-detail record datasets, originating from telecommunication organizations across the world. We statistically evaluate the effect of relational classifiers and collective inference methods on the predictive power of relational learners, as well as the performance of models where relational learners are combined with traditional methods of predicting customer churn in the telecommunication industry. Finally we investigate the effect of network construction on model performance; our findings imply that the definition of edges and weights in the network does have an impact on the results of the predictive models. As a result of the study, the best configuration is a non-relational learner enriched with network variables, without collective inference, using binary weights and undirected networks. In addition, we provide guidelines on how to apply social networks analytics for churn prediction in the telecommunication industry in an optimal way, ranging from network architecture to model building and evaluation.
\end{abstract}

Keywords: Social Networks Analytics, Churn Prediction, Relational Learning, Collective Inference, Telecommunication Industry, Network Construction

\footnotetext{
${ }^{*}$ Corresponding author

Email addresses: maria.oskarsdottir@kuleuven.be (María Óskarsdóttir), C.Bravo@soton.ac.uk (Cristián Bravo), wouter.verbeke@vub.ac.be (Wouter Verbeke), charles@grandata.com (Carlos Sarraute), bart.baesens@kuleuven.be (Bart Baesens), jan.vanthienen@kuleuven.be (Jan Vanthienen)
} 


\section{Introduction}

Customer churn prediction in telecommunication companies (telcos) has become an increasingly popular research topic in the literature in recent years (De Bock \& Van den Poel, 2011, Modani et al., 2013; Verbeke et al., 2012). The competitive landscape of these companies, in which customers have many providers to choose from and can easily switch providers should they become unhappy, creates a fierce environment that requires a high level of sophistication to thrive. Apart from that, studies have shown that customer attrition can be much more expensive for companies than customer retention is (Athanassopoulos, 2000, Berson \& Smith, 2002). A widely used strategy is therefore to identify customers with the highest propensity to churn, and offer them incentives to persuade them to stay. Long-term customers are also more profitable for the company, since they are more likely to buy additional products and spread the word of their satisfaction, thus indirectly attracting more customers (Ganesh et al., 2000). Finally, telcos gather an abundance of data about their customers, such as demographics, financials, usage behavior and call records which presents the opportunity to make these data actionable by using analytics techniques.

In classical customer churn prediction (CCP) modeling, a binary classifier is applied to available customer data at the company to build a predictive model which assigns each customer a score representing their propensity of churning. Social network analytics (SNA) has become a substantial addition to this field, as studies show that, when the customer datasets contain network features in addition to customer attributes, the performance of CCP models is enhanced (Backiel et al., 2014; Kusuma et al., 2013; Richter et al., 2010). The network features are extracted from call networks and encapsulate both calling behavior and interactions between customers. As such they carry valuable information that can be used to generate more accurate CCP models. In addition, a group of methods called relational - or network - learners have been successfully used to exploit the information flow between connected customers in a call network to predict churn (Dasgupta et al., 2008; Dierkes et al. 2011; Verbeke et al., 2014). In an exploratory study, Verbeke et al. (2014) adapted and applied the network learning framework and toolkit, NetKit, as proposed by Macskassy \& Provost (2007), to classify customers in a call network. Relational learners, which are made up of relational classifiers and collective inference methods, simulate how customers who have already churned affect others and how 'churn influence' spreads through the network. Figure 1 shows an example of a network before and after application of a relational learner. The result is a score for each customer that can be used as 

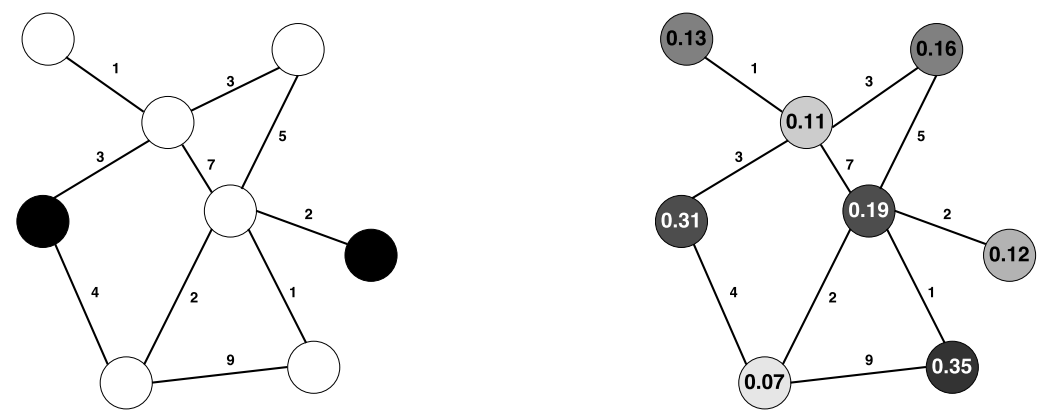

Figure 1: The figures show an example of an application of a relational learner. The figure on the left displays a graph with eight customers, of which two have churned (black) and six have not churned (white). The figure on the right shows the same network after the RL has been applied. Each customer now has a score or probability of churning.

a churn label or an additional feature in the dataset. Verbeke et al. (2014) explored both possibilities and showed that some of the relational learners had great potential when it comes to predicting churn.

When applying SNA, one of the biggest challenges is defining the social network using the available relational data. For telco, these are usually call-detail records (CDR) that need to be filtered and aggregated in an intelligent way, for which many possibilities exist. These include, but are not limited to, whether the network should be uni- or bidirectional, or whether the edges should be represented by binary weights or by weights defined by taking into account the length of the call, or the number of calls between two customers. Differentiation can be made between calls taking place on certain days or at certain times of the day and also between types of customers (retail vs. corporate). Some studies filter out connections that are non-reciprocal (Dasgupta et al., 2008, Haenlein, 2013), while others disregard phone calls that last less than a given threshold, since these possibly represent unintentional phone calls (Verbeke et al., 2014). These and many other factors need to be taken into consideration when the network is built. To the best of our knowledge, very few studies investigate the impact of how the network is defined on the techniques which are applied to them, and the resulting findings (Zhu et al., 2011; Haenlein, 2013). This could be a consequence of the fact that CDR datasets often contain millions and even billions of records which makes pre-processing and transforming them into networks both difficult and time consuming.

The objective of this study is to explore and evaluate ways of using SNA for churn prediction in telco, from network definition, to model building, and model evaluation and to discuss the implica- 
Table 1: Research Questions. Performance is measured using four measures: lift at $0.5 \%$ and 5\%, AUC, and EMP as described in subsection 3.4 .

\begin{tabular}{|c|c|c|}
\hline \multirow{4}{*}{ 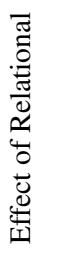 } & \multirow{4}{*}{ 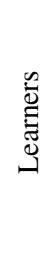 } & RQ1 Which relational learners perform statistically different from the rest when predicting customer churn? \\
\hline & & RQ2 Do some relational classifiers perform statistically better than the others? \\
\hline & & RQ3 Is the performance of some collective inference methods statistically better than the others? \\
\hline & & $\begin{array}{l}\text { When predicting customer churn, do collective inference methods improve the predictive performance of rela- } \\
\text { tional classifiers? }\end{array}$ \\
\hline \multirow{2}{*}{ 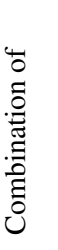 } & \multirow{2}{*}{ 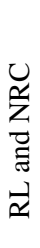 } & $\begin{array}{l}\text { Which non-relational classifier model performs best when predicting churn? A model built using network } \\
\text { RQ5 features only, a model with relational learners scores only, or a model which is built with a combination of } \\
\text { both? }\end{array}$ \\
\hline & & RQ6 Which model type performs better when predicting churn? Relational learners or non-relational classifiers? \\
\hline
\end{tabular}

tions our results have for academics and practitioners alike. To achieve this, we compare classification methods that incorporate SNA, and investigate how the network architecture affects the performance of these models. The study is composed of two main parts. Firstly, based on Verbeke et al. (2014), our goal is to rank a selection of relational learners with respect to performance and to see whether combining them with classical non-relational classifiers improves churn prediction in telco. Although Verbeke et al. (2014) observed differences in performance between the methods, they could not draw conclusions regarding the statistical significance of these differences. Therefore, we have gathered eight distinct CDR datasets from around the world, which allows us to apply statistical tests for evaluating significance of the results and provide conclusive answers. For this part of the analysis we pose six research questions as can be seen in Table 1 . The first four questions are targeted towards the relational learners with the aim of ranking them by performance when predicting churn (RQ1), as well as comparing the performance of the two components, relational classifiers (RQ2) and collective inference methods (RQ3), separately. In addition, because collective inference methods have been shown to improve the performance of relational classifiers in other fields (Jensen et al., 2004), we investigate whether this is also the case when predicting churn in telco (RQ4). Thus, we provide a detailed analysis of the various dimensions of the relational learners in terms of performance. Research question RQ5 examines whether combining relational learners with non-relational classifiers improves performance and finally, with research question RQ6 we study how relational learners perform in comparison to non-relational classifiers, and thus, whether they are good enough to be used on their own. The research questions have practical implications for practitioners in the telecommunications indus- 


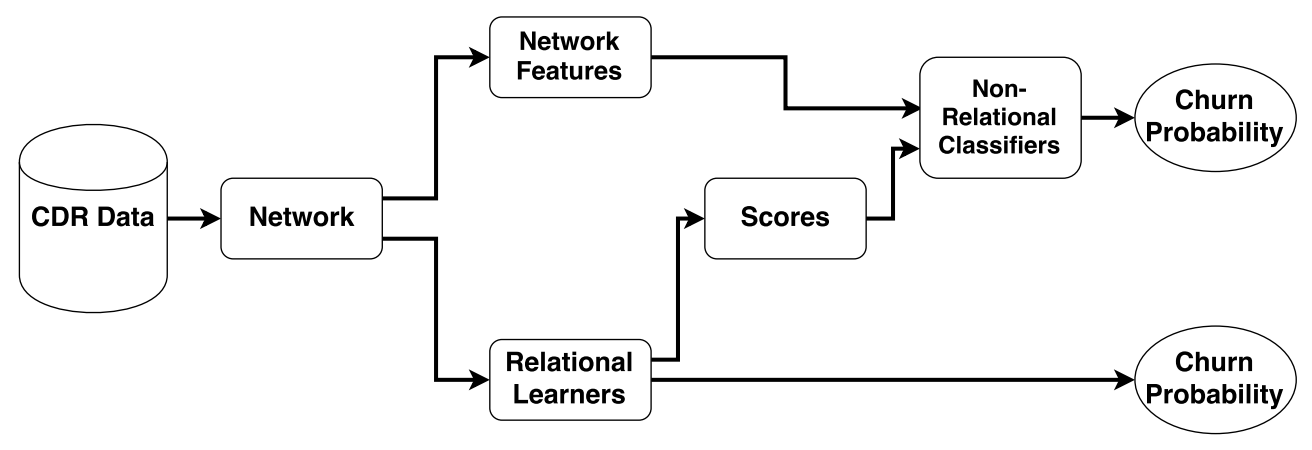

Figure 2: The model building process by exploring the impact of the network architecture or definition on model performance by studying different time frames and representation of edges and weights. Additionally, we expand the number of data sources involved, allowing us to statistically test and evaluate the results that are found and, as such, to draw conclusive answers to the above-positioned research questions. tions in Table 1 by empirically evaluating the difference in performance of a set of relational learners 
and their combination with non-relational classifiers using the eight distinct CDR datasets. Thus, we provide a ranking of the relational learners, determine whether relational classifiers perform better when used with collective inference methods, and provide conclusive answers on how to combine relational and non-relational methods to achieve optimum performance. Secondly, our follow-up study shows that the performance of CCP models does in fact depend on how the network is defined. Our results imply that the complexity of the phenomenon is not in the complexity of the weights, but in the network structure and the relationship between this structure and the other variables in the dataset.

The rest of this paper is arranged as follows. In the next section, we discuss the related literature on churn prediction in telco and network construction. Next, we describe the methodology of the current research in detail followed by a section about the experimental design that we apply. Thereafter, we present the results of the experiments together with a discussion about those results. In section 6 , we demonstrate how the model is affected by the network, and subsequently we discuss the managerial and academic insights of our results. The paper concludes with a summary of our main findings and suggestions for future research.

\section{Related Work}

As a research domain, CCP is already well established. This classification problem has been studied intensively in various sectors where maintaining relationships with current customers is considered important. It has been applied in the banking sector (Ali \& Arıtürk, 2014; Glady et al., 2009; Larivière \& Van den Poel, 2004; Van den Poel \& Lariviere, 2004; Xie et al., 2009), by insurance companies (Guillén et al., 2012, Günther et al., 2014), internet service providers (Khan et al., 2010), online social networks (Ngonmang et al., 2012), and in the telecommunication industry (Chen et al., 2012), which is the case we discuss here. We refer to Verbeke et al. (2012) for an overview of commonly used classification techniques for $\mathrm{CCP}$ in telco and a benchmarking study of those techniques.

Recent publications where social network analysis has been applied to predict customer churn in telco can be seen in the top part of Table 2. These studies show how the performance of CCP models is enhanced when network effects are taken into account by means of SNA. The table also lists how edges and weights are defined in each case, together with the number of nodes in the networks and the duration of the CDR data that are used in each study.

As Table 2 explains, the most widely used relational learner is the spreading activation method (Dasgupta et al., 2008). It is used on its own (Backiel et al., 2015; Dasgupta et al., 2008) and to 


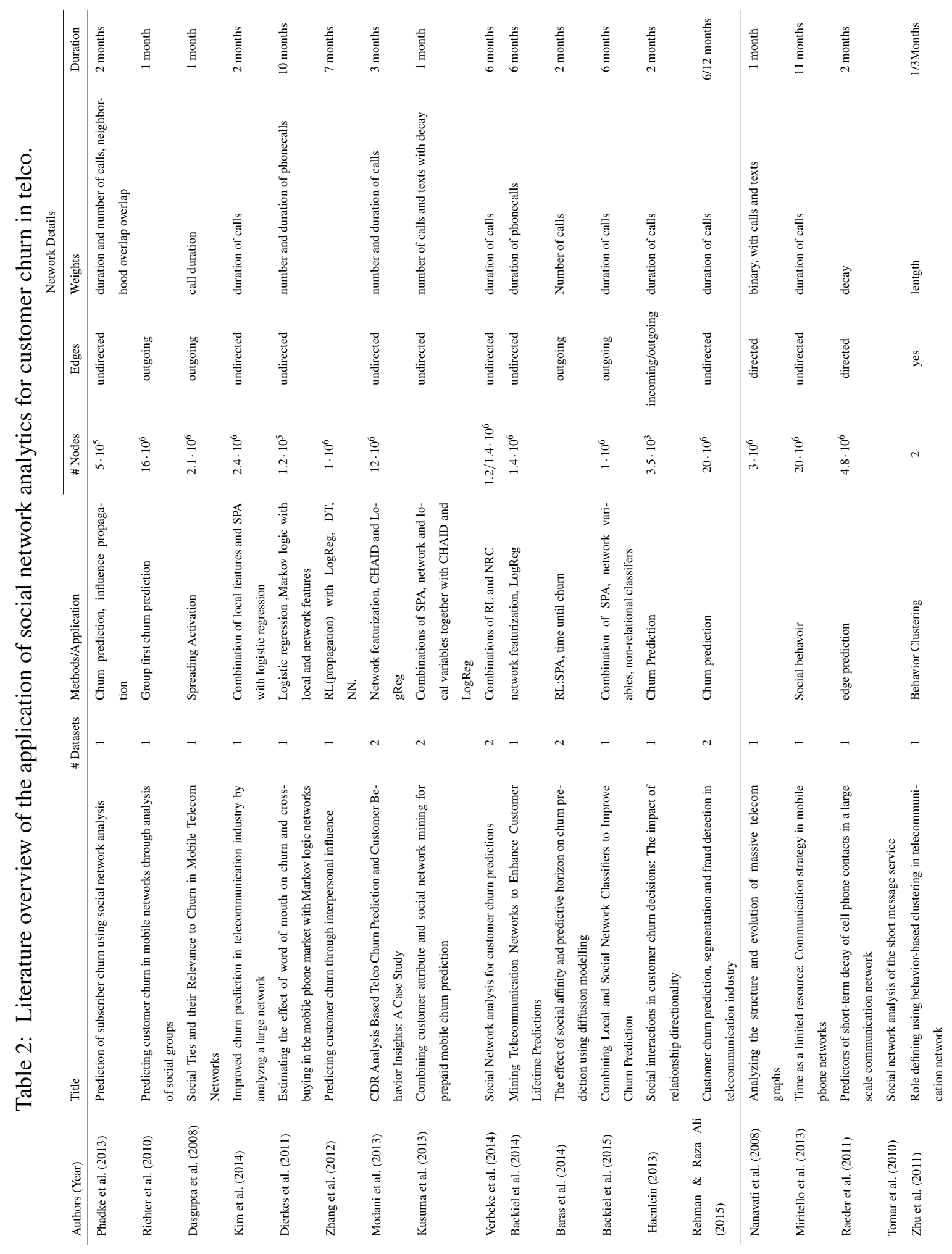


produce scores that are then used as variables in non-relational classifiers, such as logistic regression and decision trees (Kim et al., 2014, Kusuma et al., 2013). Other works enrich their datasets with network measures for non-relational classifiers, instead of exploiting relational learners (Backiel et al., 2014; Benedek et al., 2014; Modani et al., 2013; Zhang et al., 2012). Verbeke et al. (2014) compare the performance of multiple relational learners to the performance of non-relational classifiers, with and without network variables. The best model is obtained when predictions from both relational learners and non-relational classifiers with network variables were combined. Additionally, Backiel et al. (2015) combine these types of models in various ways, with the result that the combined model, a binary classification built using the scores resulting from non-relational classifiers and relational learners (in this case SPA) as variables, gives the best result. Contrary to the current study, almost none of the discussed papers include a comparison of relational learners in terms of performance or apply their techniques to multiple CDR datasets.

For most of the papers in Table 2, SNA relies on a single network which is defined and constructed only once for the situation at hand. In most cases, length of phone calls between customers are used as weights, but the directionality of the edges varies. All studies rely on in-network customers only, since information about customers of other telcos is rarely available.

Only a handful of studies compare the effects of networks defined in different ways or test the difference in results obtained from different networks as we do here. For example, Haenlein (2013) studies the dynamics of social interactions for customer churn within a directed network. He compares customers' propensity to churn using call networks with incoming calls, outgoing calls and both together, producing an undirected network. According to his study, a customer is more likely to churn if they have been in contact with a person who already churned, but only if the relationship is outgoing, meaning that the customer calls the churned person, and not the other way around. In addition, Zhu et al. (2011) apply behavior-based clustering to define roles of the customers in their call network using both incoming and outgoing edges. The people they identify as potential churners make many phone calls but have low betweenness and closeness. In addition, the length of incoming and outgoing calls is higher than for other customers.

So far we have only discussed papers that exploit CDR data for churn prediction in telco, whereas numerous other applications exist. An extensive survey by Naboulsi et al. (2015) on the fast-growing field of mobile traffic analysis and how CDR data are increasingly used in data-mining applications classifies the analyses in the existing literature as social, mobility, and network analyses. Some of 
the research that falls into the first of these classes, which deals with human dynamics and social interactions, pays special attention to network construction. The results of these studies might possibly have interesting implications for problem-setting in our context. The lower part of Table 2 contains a brief overview of papers where CDR data have been used to build networks for SNA in telco with various objectives.

Miritello et al. (2013) examine how callers distribute their time across their social network in relation to their network size and intensity of mobile use. According to their results, people with more connections spend more time communicating than people with fewer connections and the average time spent on each connection increases as the number of connections increases, up to a threshold of 10 to 40 connections. In addition, independent of the size of their network, people distribute their time unevenly, thus dedicating a small amount of time to many people and a great deal of time to a few people. In a study about the decay of edges in a call network, Raeder et al. (2011) use weighted data to determine the importance of edge weight for the persistence and decay of connecting between people in a call network. Using machine learning techniques, their results imply that directed edge weight, reciprocated edge weight and recency of connections are important features when predicting edge decay.

Park et al. (2012) propose a relational learner, similar to the spreading activation algorithm, in order to to validate self-reported demographic data of customers. In their network construction they use out-degree, number of outgoing calls and duration of outgoing calls and the results imply that the best performing models are those that use networks with out-degree.

Featurizing networks offers the possibility to compare the importance of various network definitions. This was the case in Sarraute et al. (2015) who extract features based on different edge and weight definitions, such as incoming and outgoing calls, length and number of calls, and number of text messages in addition to taking time of day and the day of week into account. In a subsequent PCA analysis, total number of calls, total duration of calls, and total number of text messages are shown to explain most of the variance. This indicates that the activity of users is a good candidate to characterize users' social behavior. 


\section{Customer Churn Prediction Using Social Networks Analytics}

\subsection{Networks}

In this particular predictive analytics framework, SNA-based methods and binary classifiers are applied to assign each customer of a telco to one of two classes: churner or non-churner. The starting point of conducting social network analysis is the network itself (Newman, 2010). A network is composed of nodes and edges, which in this case are the customers of a telecommunications operator and the correspondence between them, respectively. Formally, the node component $\mathbf{V}$ of the network consists of a set of nodes $\mathcal{V}=\left\{v_{1}, \ldots, v_{n}\right\}$ and a label vector $\mathcal{L}=\left\{l_{1}, \ldots, l_{n}\right\}$ where each $l_{i} \in \mathcal{C}=$ $\left\{c_{1}, \ldots, c_{m}\right\}$ is the class label -in this case churner or non-churner- of node $v_{i}$. We denote by $\mathcal{V}^{K}$ and $\mathcal{V}^{U}$ the sets of nodes for which class labels are known and unknown, respectively. The known labels are used to infer labels for the unknown ones.

The edge component $\mathbf{E}$ of the network consists of two parts, edges and weights. The edges, $\mathcal{E}$, are a set of the two-subsets of $\mathcal{V}$, where the edge $e_{i j} \in \mathcal{E}$ represents an existing connection from $v_{i}$ to $v_{j}$, i.e. if the two customers have shared a phonecall. If an edge exists between nodes $i$ and $j$ we say they are connected, and if

$$
\forall i, j \in 1, \ldots, n: e_{i j} \in \mathcal{E} \Longleftrightarrow e_{j i} \in \mathcal{E}
$$

we say that the network is undirected and directed otherwise. Incoming and outgoing edges of a node $v_{i}$ are the sets of nodes of the form $e_{j i}$ and $e_{i j}$, respectively

A non-negative weight, $w_{i j}$, can be associated to each edge to denote the strength of the connection. There are various ways to define the weights. They can be binary, indicating whether two nodes are connected or not, or be assigned some other value based on additional information. In addition, weights can vary in time by conferring more recent connections higher importance than older connections. To model this in the network, the weights at time $t, w_{i j, t}$ can be exponentially weighted in time by

$$
\left(w_{i j}\right)_{t}=e^{-\gamma t} w_{i j, t}
$$

where $\gamma$ is the decay constant. We obtain the final weights by aggregating all $\left(w_{i j}\right)_{t}$ for the whole time period. Weighted networks have been successfully used in credit card and social security fraud detection (Van Vlasselaer et al., 2015, 2016). 
Finally, the first order neighborhood $\mathcal{N}_{i}^{1}$ of a node $v_{i} \in \mathcal{V}$ is the set of nodes that are connected to $v_{i}$

$$
\mathcal{N}_{i}^{1}=\left\{v_{i}\right\} \cup\left\{v_{j} \mid e_{i, j} \in \mathcal{E}, j=1, \ldots, n\right\}
$$

\subsection{Enriching Non-Relational Classifiers with Network Features}

A network can be used to extract network features in a process called featurization (Baesens et al.

2015). Information from the neighborhood of each node, such as the labels of connected nodes and weights of the edges between them, is used to compute numerical attributes to characterize and describe the nodes. Examples of such features include the number of neighbors a node has (degree), the number of fully connected subgraphs of three nodes (triangles), relational neighbor score, and probabilistic relational neighbor score. Various features describing the position and connectivity of a node within a network also exist (Newman, 2010).

Link-based features are a specific group of network features, introduced by Lu \& Getoor (2003). These features are constructed using class labels, in addition to nodes and edges. They define three types of features: mode-link, count-link and binary-link. The first one, mode-link, is a single feature defined as the most frequently occurring class label amongst the nodes in the neighborhood. The count-link statistic counts the number of times each different class label appears in the neighborhood, and the last statistic, the binary-link, documents for each class in $\mathcal{C}$ whether or not it appears as a class label for a node in the neighborhood.

Featurization has been successfully used to improve the performance of churn prediction models in telco; see Table 2. Typically, the extracted network features are added to the customer dataset, before binary classifiers, such as decision trees, logistic regression, support vector machines and artificial neural networks are used to train and test CCP models using the enriched dataset. We limit the number of binary classifiers, since our goal is not to compare them. Instead, we choose three that have been shown to perform well when predicting churn in telco (Verbeke et al. 2012). We choose the classifiers logistic regression (Log) (Coussement \& Van den Poel, 2008; Mozer et al., 2000), random forests (RF) (Anil Kumar \& Ravi, 2008), and artificial neural networks (NN) (Hung et al., 2006) because they represent different aspects of the trade-off between complexity and predictive capability (Verbraken et al. 2014). Popular in the industry, logistic regression is simple to understand, whereas NN and RF are more powerful but harder to interpret since they are black-box models. In the model building we refer to these classifiers as non-relational classifiers (NRC). Alternative binary classifiers were 

work while taking into account how the inferred labels affect each other. They decide in which order the nodes are labeled and how a final label is determined. They have been shown to improve the performance of relational classifiers in genomes and bibliographic networks (Jensen et al., 2004, Sen et al., 2008). In general, CIs perform two operations iteratively until a terminating condition is met. used to update the labels of the nodes. The methods used here are Gibbs sampling (Gibbs), iterative classification (IC), relaxation labeling (RL), relaxation labeling with simulated annealing (RLSA), and

\footnotetext{
${ }^{1}$ The electronic companion is submitted as supplementary material.
} 

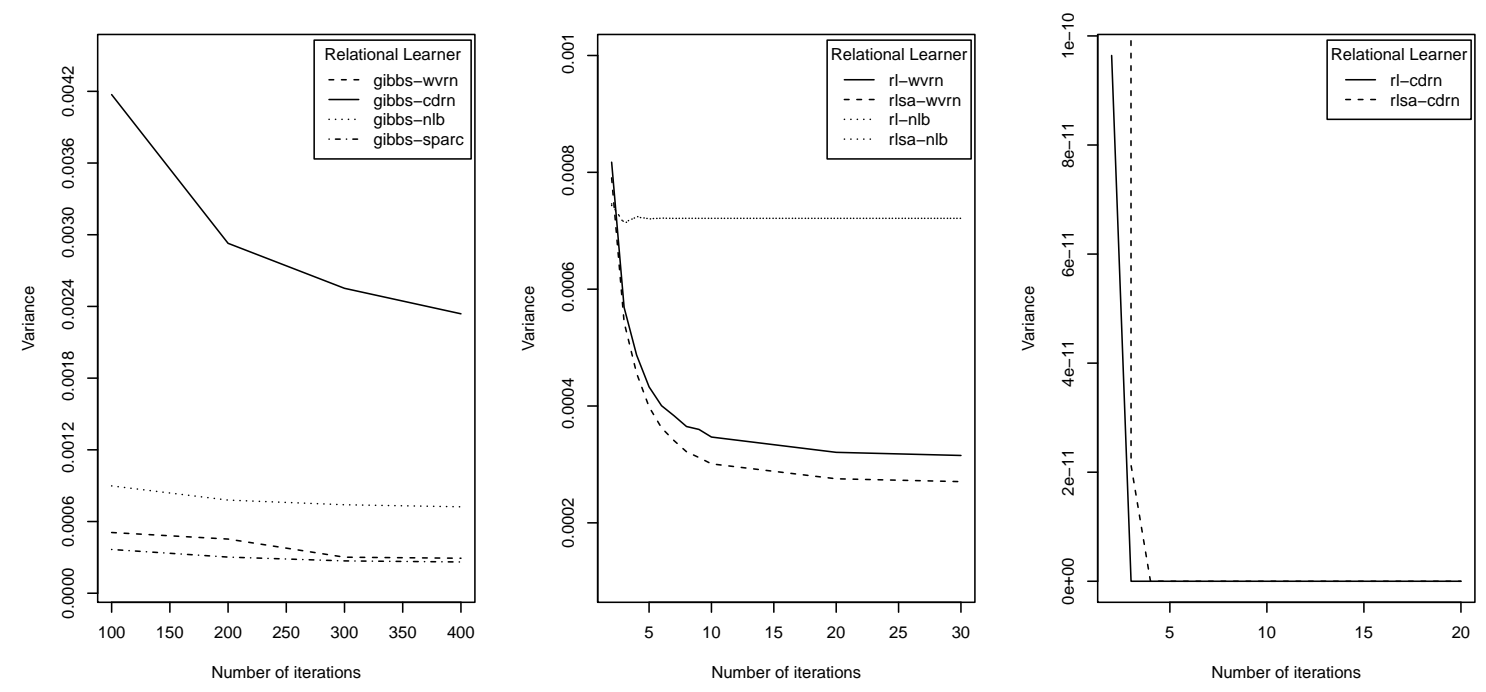

Figure 3: The figures show the results of the sensitivity analysis of some of the relational learners.

spreading activation collective inference method (SPA CI). We refer to the electronic companion for descriptions and pseudo codes for the CI methods.

When CI methods iteratively classify the nodes in a network, a smoothing effect of the churn influence may occur. Before benchmarking the methods along all available datasets, we investigate this effect for each of the relational learners using one of the datasets. Figure 3 shows the variance of the predicted churn scores as a function of the number of iterations in the collective inference method for various relational learners. This sensitivity analysis shows that the variation decreases very rapidly as the number of iterations increases. This means that, with the default settings of each CI method, the variation of the final scores is very low and consequently there is very little distinction between churners and non-churners. In particular, for the iterative classification CI, the scores stabilize after only a few iterations. It can be seen from the figure, that for some relational classifiers, such as WVRN, this decline in variance happens faster than for the others. We note that the CI spreading activation was not included, as it already contains an early stopping mechanism to compensate for this kind of smoothing effect. Based on the sensitivity analysis, it was decided to implement the same kind of mechanism for early stopping in the other CIs.

Overall, we consider four relational classifiers and five collective inference methods, which, together with the option of not applying a CI, results in a total of two dozen unique combinations of 
methods, or 24 relational learners.

\subsection{Performance Measures}

We next discuss two commonly used measures - lift and AUC - in addition to two more recent ones - the Maximum Profit and the Expected Maximum Profit measures. The first two measures are applicable in classification problems, whereas the last two are specifically developed for churn prediction models.

Lift is a commonly used performance measure for customer churn (Hung et al., 2006; Tufféry, 2011; Verbeke et al., 2014). It compares the ratio of churners in a fraction of customers with the highest predicted probabilities to the ratio of churners in the actual customer base. Thereby, it represents how much better a prediction model is at identifying churners, compared to a random sample of customers. The top decile lift, considering the top $10 \%$ of the customer base, is typically used. However, this choice of the top fraction is arbitrary and does not necessarily reflect the needs of the company, especially if the customer base is large, as is the case for some of the datasets in this study, see table 3 . Including too many customers in a retention campaign does not only increase the cost of the campaign but also increases the risk of offering promotions to customers who have no intention of churning. Therefore, it is more meaningful to consider smaller fractions to identify only those who are most likely to churn. To compensate for the variation in number of observations in our datasets we choose to use lift at $0.5 \%$ and $5 \%$. As a result, the lift measure proves meaningful for both large and small organizations.

Another widely used and well established performance measure is the receiver operating characteristic curve (ROC) and its corresponding AUC value (Fawcett, 2006). AUC is typically a number between 0.5 and 1 and encapsulates the trade-off between the true and false positive rates. It can be interpreted as the probability that a randomly chosen churner is ranked higher than a randomly chosen non-churner. Recent studies have suggested Hand's H-measure as a coherent alternative to the AUC measure (Hand, 2009) but because of evidence of correlation between these two (Lessmann et al. 2015), we will only include the widely used AUC.

In a business setting, $\mathrm{CCP}$ modeling is usually conducted to decide which customers to target in a retention campaign. As such, the specific requirements of the campaign should be taken into account when evaluating the models, to accurately measure the anticipated profit. The maximum profit measure (MP; Verbeke et al., 2012) was developed with this objective. Based on the total profit of a 
retention campaign, as proposed by Neslin et al. (2006), the average classification profit for customer churn is defined as

$$
P^{c c p}(t ; \gamma, C L V, \delta, \phi)=C L V(\gamma(1-\delta)-\phi) \cdot \pi_{0} F_{0}(t)-C L V(\delta+\phi) \pi_{1} F_{1}(t)
$$

with $\eta$ the fraction of customers that are targeted, $\gamma$ the probability that a customer accepts the incentive, $C L V$ the average customer lifetime value, $\delta$ the cost of the incentive, $\pi_{0}$ the base churn rate and $\pi_{1}$ the base non-churn rate, $F_{0}(t)$ and $F_{1}(t)$ the cumulative density functions for churn and non-churn, respectively, given the cut-off $t, \lambda$ the percentage of churners within the targeted fraction, and $\phi$ the cost of contacting the customer. The maximum profit $(M P)$ measure is then defined as

$$
M P^{c c p}=\max _{\forall t} P^{c c p}(t ; \eta, \gamma, C L V, \delta, \phi)
$$

and represents the fraction of customers that should be targeted for a campaign to achieve maximum profit. As many of the parameters are not always known, a corresponding expected maximum profit $(E M P)$ measure (Verbraken et al., 2013) is proposed. It is given by

$$
\left.E M P^{c c p}=\int_{\gamma} P^{c c p} T(\gamma) ; \gamma, C L V, \delta, \phi\right) \cdot h(\gamma) d \gamma
$$

where $T$ is the optimal cut-off value for a given $\gamma$ and $h(\gamma)$ is the probability density function for $\gamma$, chosen as the beta distribution as in the $H$-measure.

The maximum profit measure has advantages over both the lift measure and AUC. Firstly, the fractions chosen for the lift measure are arbitrary and take neither costs nor benefits into account when measuring the performance. While both lift and AUC are universal and applicable to any classification problem, MP and EMP are the only measures that are specifically designed to take into account the requirements of a CCP model, which is the campaign itself, its costs, and its expected return. Here, we choose to use the EMP measure because it is more robust in terms of the inherent stochastic nature of costs and benefits in churn management.

\section{Experimental Design}

\subsection{Data Description and Preparation}

Following collection, eight distinct CDR datasets were analyzed in order to provide answers to the research questions in Table 1 . Table 3 summarizes the main features of the datasets. Most of the data come from telcos in Europe, while some have their origins in North America. 
Table 3: Dataset Description

\begin{tabular}{llllll}
\hline ID & Origin & Year & Customers & Churn Rate & Sparsity \\
\hline BC1 & Belgium & 2010 & $1.41 \cdot 10^{6}$ & $4.4 \%$ & $7.93 \cdot 10^{-7}$ \\
BC2 & Belgium & 2010 & $1.21 \cdot 10^{6}$ & $0.84 \%$ & $2.20 \cdot 10^{-6}$ \\
GD1 & North America & 2015 & $1.57 \cdot 10^{6}$ & $0.71 \%$ & $3.14 \cdot 10^{-6}$ \\
GD2 & North America & 2015 & $1.32 \cdot 10^{6}$ & $2.5 \%$ & $1.69 \cdot 10^{-6}$ \\
BP1 & Europe & 2009 & $4.33 \cdot 10^{6}$ & $8.5 \%$ & $9.42 \cdot 10^{-7}$ \\
BP2 & Europe & 2008 & $4.52 \cdot 10^{6}$ & $3.5 \%$ & $9.44 \cdot 10^{-7}$ \\
MV & Belgium & 2012 & $1.70 \cdot 10^{5}$ & $8.3 \%$ & $1.86 \cdot 10^{-5}$ \\
IS & Iceland & 2015 & $9.36 \cdot 10^{4}$ & $2.3 \%$ & $1.04 \cdot 10^{-4}$ \\
\hline
\end{tabular}

All datasets contain six consecutive months of cell phone usage data for the customers of the therefore excluded from the analyses. To ensure comparability of the different types of models, the fifth month is always used as the test and prediction month. As a result, the first four months of each 
dataset are used to build the models. The fraction of churners in the customer base, or the churn rates, two levels each, there are four different combinations of the two factors.

The relational learners and the non-relational classifiers require different setups for conducting the 


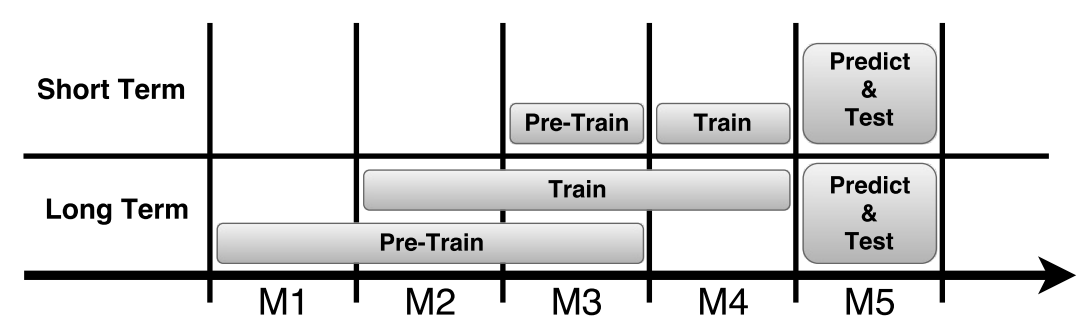

(a) Relational Learners

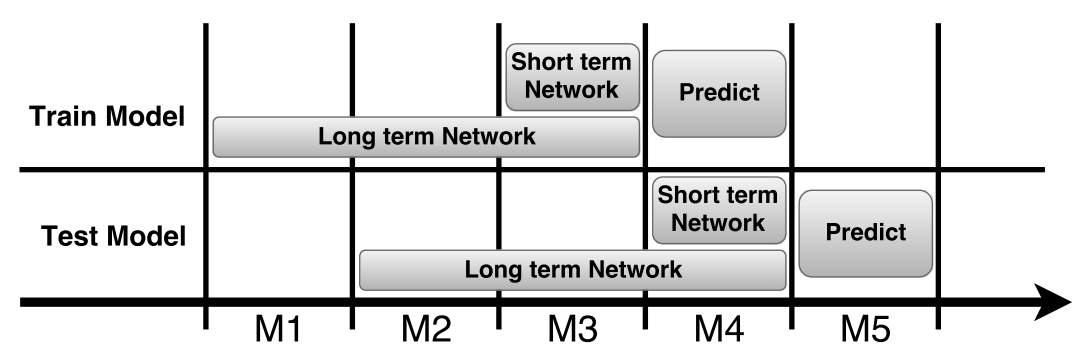

(b) Non-Relational Classifiers

Figure 4: The figures show how the datasets are split up by month to build the networks for pretraining, training and predicting in the short- and long-term settings for the relational learners and for out-of-time testing for the non-relational classifiers.

experiments, as we describe below.

\subsubsection{Setup for Relational Learners}

In case of relational learners, our objective is to rank all two dozen of them, as well as evaluate the RC and CI separately. We implement the time aspect of CCP as in Verbeke et al. (2014), that is, we train the methods at a specific time, $t$, where all labels are known, and use the resulting scores as the estimated labels at time $t+1$, which in this case equals $M 5$. This means that we assume that $\mathcal{V}^{K}=\emptyset$ at time $t+1$ before the analyses are undertaken.

As described in the online companion, two of the relational learners, CDRN and NLB, need a pretraining period, defined as having the same length as the corresponding training period, but starting one month prior as in Verbeke et al. (2014). In summary, eight networks are built for the analysis of each dataset: for each weight representation, there are two short-term networks, $M 3$ and $M 4$, and two long-term networks, $M 1$ to $M 3$ and $M 2$ to $M 4$. This is depicted in Figure $4 \mathrm{a}$.

Overall, each RL will produce four sets of scores for each of the eight datasets, or a total of 32 scores. 
As is evident from Table 3 there is severe imbalance in the distribution of the two classes, churners and non-churners. Because of the networked nature of the data, commonly used sampling techniques,

\section{Relational and Non-Relational Learner Effects}

In this section, we present and discuss the results of the experiments. We follow the guidelines of (Demsar, 2006) to statistically compare classifiers and methods. More precisely, to measure a differ-

\footnotetext{
${ }^{2}$ Network features include degree, full, churn and non-churn; triangles, full, churn and non-churn; transitivity and linkbased measures, described in subsection 3.2
} 
ence in performance of various methods, a Friedman test is applied to the average rank in performance difference in performance between all RC, measured by $0.5 \%$ lift. The gray boxes, which represent significant differences, show that the differences NLB-CDRN, spaRC-NLB and WVRN-NLB are all 


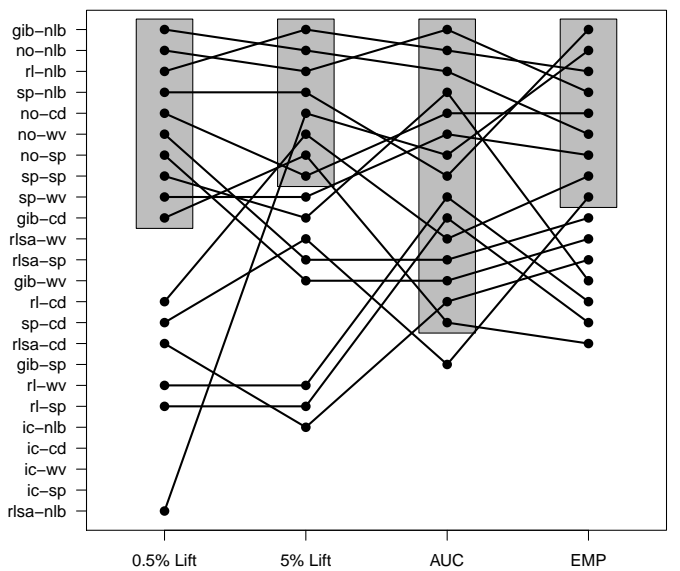

Figure 5: The figure shows the relative ranks, from best, at the top, to worst, at the bottom, as measured by the four performance measures. The gray boxes represent the methods which are not significantly different at the $95 \%$ confidence level. Lines for the methods which performed significantly worse by all measures were not drawn.

significant. This implies that NLB performs significantly better than all the other relational classifiers, and it is the only one that differs from the rest. Similar figures for the other performance measures show the same results. The superiority of NLB can be explained by how comprehensive it is, as it considers the whole network before inferring labels. In a pre-training step, this classifier builds a logistic regression model for the nodes in the network, using their link-based measures. This model is then used in the inferencing process, and because it already has internalized the information from the whole network it is able to make more accurate predictions. In contrast, the other relational classifiers calculate a score based on each node's neighborhood, without any pre-training and without taking into account the rest of the network.

The collective inference methods are tested in the same way. Again the Friedman test results in low enough p-values and the results of a post-hoc analysis of the differences can be seen in Figure $6 \mathrm{~b}$. The results in the figure are based on the $0.5 \%$ lift, but performance according to the other measures shows the same behavior. As the figure shows, IC always performs significantly worse than the other methods, and not applying CI performs significantly better than applying any of the CIs. A probable explanation for why IC performs worse than the rest is that, in each step of the iteration, it 


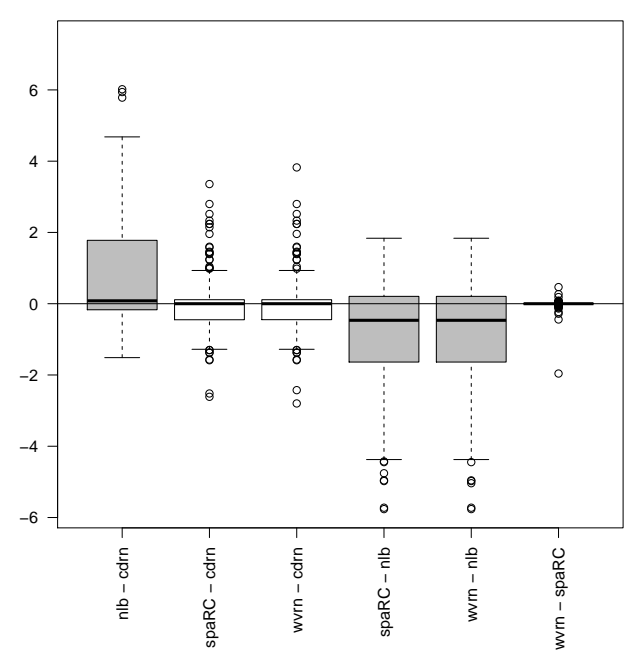

(a) Differences of Relational Classifiers

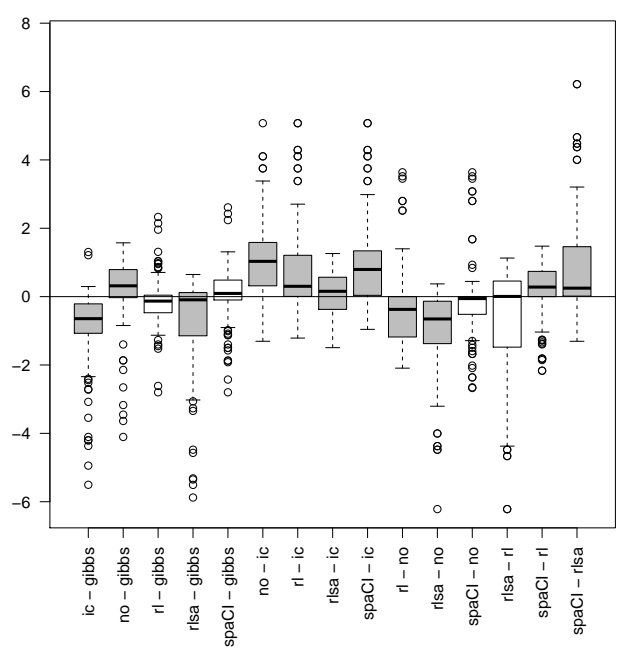

(b) Differences of Collective Inference Methods

Figure 6: The figures show pairwise differences in performance measured by $0.5 \%$ lift of the four relational classifiers (left) and the six collective inference methods (right). Gray- and white- colored boxes represent differences that are and are not significant at the $95 \%$ confidence level, respectively.

assigns churn labels and not probabilities or scores like the other methods. By making such absolute decisions, a significant amount of information is lost, and there is less flexibility when inferencing in subsequent steps; therefore the prediction-making process becomes less accurate on the whole. The sensitivity analysis in subsection 3.3 even reveals that the assigned labels do not change after the first few iterations.

Finally, we test whether there is a difference in performance between methods with CI and without CI using the parametric Kruskal-Wallis test. The p-value for each performance measure was less than 0.001, meaning that there is strong statistical evidence of the difference between methods that apply $\mathrm{CI}$ and methods that do not. Further investigation shows that methods without CI performed better. Although this may be a contradictory result to previous findings on the performance of collective inference methods (Jensen et al., 2004; Sen et al. 2008), there are various possible explanations for this behavior. In fact, as we have already shown, the variation in churn scores decreases very rapidly when CIs are applied, thus decreasing the predictive performance. The call networks in this specific problem setting are very large and sparse, and with few churn signals. As a result, when the CIs start spreading the 'churn influence' it gets too diluted and in the end the signals are not strong enough or 
too similar to the non-signals to be meaningful. This also seems to suggest that 'churn influence' does learner scores do possess valuable knowledge that is not captured by network features alone. This is further confirmed by the fact that the scores were almost never correlated with the network features, although there were often correlations within each set of features. However, scores produced when 


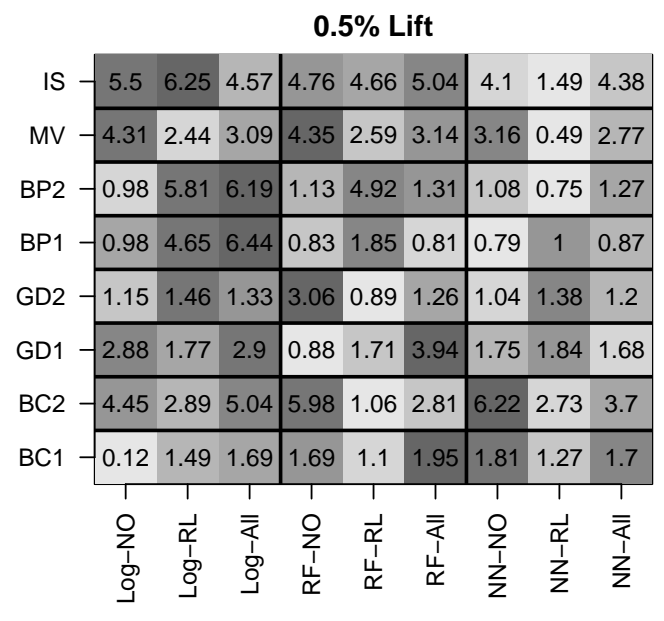

AUC

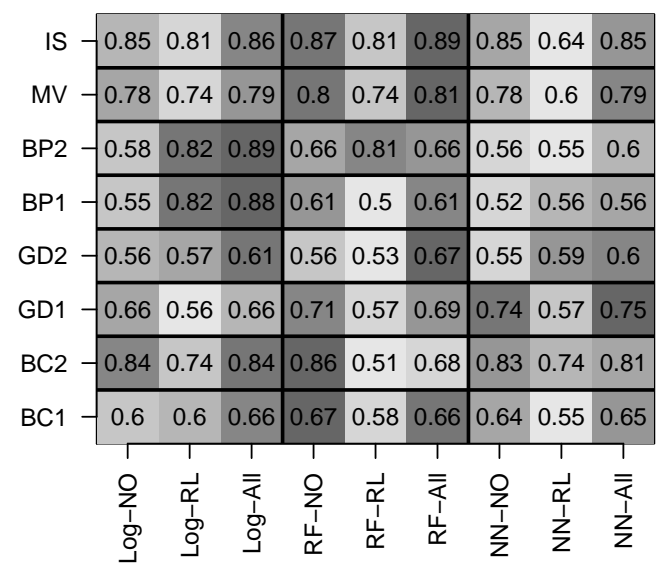

$5 \%$ Lift

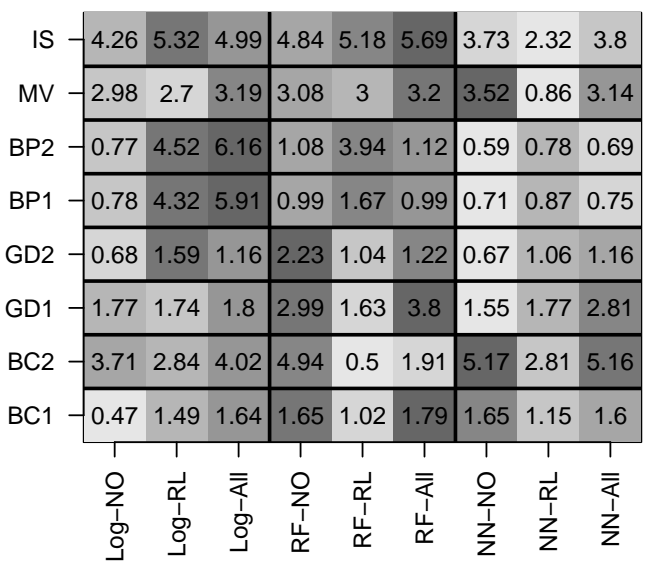

EMP

\begin{tabular}{|c|c|c|c|c|c|c|c|c|c|}
\hline IS & 4 & 7 & 8 & 5 & 6 & 9 & 2 & 1 & 3 \\
\hline MV & 5 & 2 & 6 & 8 & 3 & 9 & 4 & 1 & 7 \\
\hline BP2 & 2 & 8 & 9 & 2 & 4 & 2 & 6 & 5 & 7 \\
\hline BP1 & 5 & 8 & 9 & 2 & 7 & 3 & 1 & 6 & 4 \\
\hline GD2 & 8 & 2 & 6 & 9 & 2 & 7 & 2 & 4 & 5 \\
\hline GD1 & 8 & 3.5 & 9 & 3.5 & 3.5 & 7 & 3.5 & 3.5 & 3.5 \\
\hline $\mathrm{BC} 2$ & 9 & 6 & 8 & 2.5 & 2.5 & 7 & 2.5 & 5 & 2.5 \\
\hline $\mathrm{BC} 1$ & 3 & 4 & 6 & 5 & 2 & 9 & 7 & 1 & 8 \\
\hline & $\begin{array}{l}0 \\
\sum_{1} \\
0 \\
0\end{array}$ & $\begin{array}{l}\vec{\sim} \\
\vdots \\
\delta \\
\delta\end{array}$ & $\begin{array}{l}\bar{z} \\
\vdots \\
0 \\
\end{array}$ & 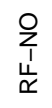 & $\begin{array}{l}\overrightarrow{\widetilde{r}} \\
\underline{\underline{u}} \\
\widetilde{\sim}\end{array}$ & $\begin{array}{l}\overline{\bar{\tau}} \\
\underline{\sim} \\
\widetilde{\alpha}\end{array}$ & $\begin{array}{l}\text { O } \\
\sum_{1} \\
\sum_{z}\end{array}$ & $\begin{array}{l}\frac{1}{\alpha} \\
\frac{1}{z}\end{array}$ & $\begin{array}{l}\bar{z} \\
\overline{1} \\
\bar{z}\end{array}$ \\
\hline
\end{tabular}

Figure 7: The figures compare the performance of each of the three non-relational classifiers with the three subsets of variables for the eight datasets and the four performance measures. Each row represents one dataset. In the first three columns on the left are the logistic regression models $(\log )$, in the three columns in the middle are the random forests models (RF) and in the last three columns on the right are the neural networks models $(\mathrm{NN})$. Each classifier is used to build a model using network variables only (NO), RL scores only (RL), and network variables together with RL scores (All). The figures show the the measurement values -or the actual rank in the case of EMP- of each combination and a color coded ranking of the nine models for each dataset, with a darker color representing a better performance. 
Table 4: P-values from the Kruskal-Wallis test when comparing the difference in performance between each relational learner listed in the table and all non-relational classifiers.

\begin{tabular}{lrrrr} 
& \multicolumn{4}{c}{ Performance Measure } \\
\cline { 2 - 5 } Relational Learner & $0.5 \%$ lift & $5 \%$ lift & AUC & EMP \\
\hline gibbs-nlb & 0.47 & 0.65 & $<0.001$ & 0.70 \\
no-nlb & 0.98 & 0.50 & $<0.001$ & 0.64 \\
rl-nlb & 0.60 & 0.34 & $<0.001$ & 0.81 \\
spa-nlb & 0.60 & 0.70 & $<0.001$ & 0.81 \\
no-cdrn & 0.004 & $<0.001$ & $<0.001$ & 0.22 \\
no-wvrn & 0.003 & $<0.001$ & $<0.001$ & 0.16 \\
no-spaRC & 0.003 & $<0.001$ & $<0.001$ & 0.16 \\
spa-sparc & 0.002 & $<0.001$ & $<0.001$ & 0.071 \\
\hline
\end{tabular}

applying the CI Gibbs sampling were never correlated with any other features and not even amongst themselves, which was a common behavior for the other RL scores.

We can conclude, based on our results, that models with a combination of network features and relational learner scores perform best (RQ5).

\subsection{Comparison of $R L$ and $N R C$}

Regarding research question RQ6, when comparing the predictive performance of relational learnperformance of each relational learner, that appears in the gray box for lift at 5\% in Figure 5, to the overall performance of the three non-relational classifiers. The resulting $\mathrm{p}$-values for the four performance measures can be seen in Table 4 . The table shows that the first four relational learners are only 


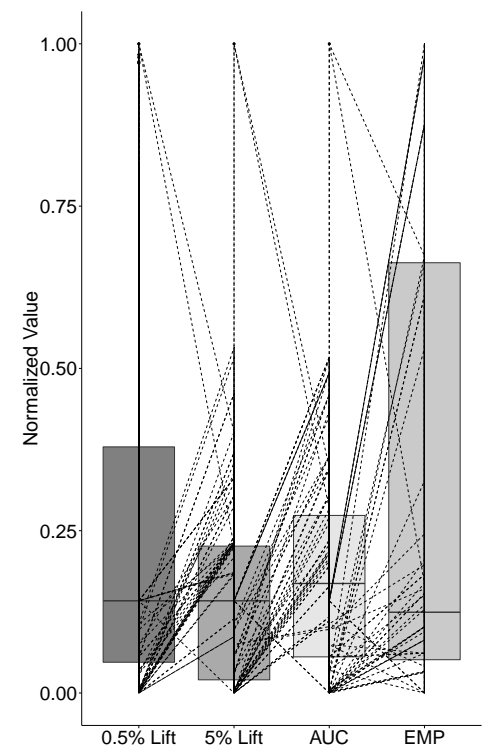

(a) Relational Learners

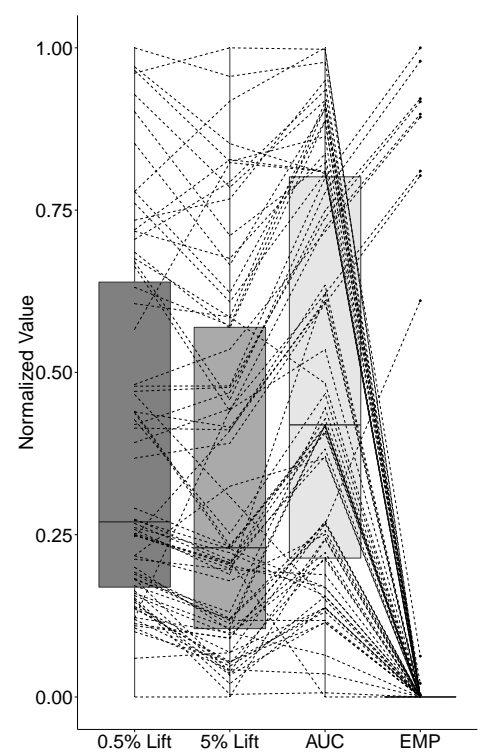

(b) Non-Relational Classifiers

Figure 8: The figures show, for the relational learners (left) and for the non-relational classifiers (right), the distribution of the four performance measures (boxplots) and the correlations between them (dotted lines).

outperformed by the non-relational classifiers when performance is measured using AUC, but not using any of the other three performance measures. The last four relational learners are all outperformed by the non-relational classifiers, except when measured using EMP.

We conclude our discussion of relational learners and non-relational classifiers by looking at their distribution and correlations, see Figure 8 . For the two approaches, we normalize the values of each performance measure in order to make them comparable and present their distributions with boxplots. Subsequently, measurements of the same model are connected, as shown by the dotted lines. The figure thus illustrates a combination of box- and scatter- plots with correlations. The distributions of the measures vary, as the box plots show, and the values are also not very correlated, as illustrated by how frequently the dotted lines connecting the measurements intersect. Since all the measures give consistent results, this is a good indication of the robustness of our findings. Note that in Figure $8 \mathrm{~b}$, most of the values of the EMP measure are very small, whereas one of the datasets resulted in values that were much higher (by a factor of $10^{6}$ ). This explains why there is a cluster around 0 in the figure.

To answer the final research question, we assert that overall, non-relational classifiers perform 
better than relational learners, although a few of them are just as good at predicting churn as the non-relational methods.

\section{Effects of Network Construction}

As mentioned, research on the exact impact of network construction on models and findings in the telecommunication industry is scarce. In most studies, a network is built in a specific way, often without detailed supporting arguments or explanations (Dasgupta et al. 2008). A construction issue which is overlooked in such studies is that the findings could depend on how the network is defined, and might therefore change if the network is constructed differently by whoever is conducting the experiment. Clearly, such a situation would result in potentially incorrect conclusions, as the predictive capability of the model would not be affected by the sheer explanatory power of the data but by the decisions of the modeler.

Here, in a follow-up study, we explore this potential effect, by building more than 500 networks with varying definitions of edges and weights and aggregating them in numerous ways. The detailed setup to arrive at the 500 networks will be explained in subsection 6.1. The number of networks can increase very rapidly when taking all possibilities into account, and as a result, it becomes computationally infeasible to compare all model combinations. Instead, we exploit the result of the benchmarking study in the previous section for 'algorithm selection'. Since we have shown that CIs do not improve the performance of RC, those RLs can be left out, and based on Table 4 some RLs are just as good at predicting churn as NRC is. Therefore, we have chosen one relational learners (NLB) as a proxy method to predict churn in the different types of networks to compare the performance of the resulting models. As a result, we hope to guide further studies on how to optimize network architecture when predicting churn in telco.

\subsection{Defining the Network Architecture}

For this follow-up study, we select the BC2 dataset as it represents an 'average' among all the datasets, both in size and sparsity.

As before, predictions are made for month $M 5$ and, to reduce complexity, the relational learner was applied on one time frame - the short-term time frame which spans one month. Since NLB needs a pre-training period as well, months $M 3$ and $M 4$ are used for the pre-training and training, respectively. As in section 4.1, phone calls lasting less than four seconds are removed. The edges of 
Table 5: Segmentation of Networks

\begin{tabular}{cllll}
\hline Day & Part-of-Week & Time-of-Day & Comb. Part-of-Week & Comb. Time-of-Day \\
\hline Monday & Working days(WD) & Day(8-16) & $\frac{1}{2} \mathrm{WD}+\mathrm{WE}$ & $\frac{1}{2}$ Day+Evening \\
Tuesday & Weekend(WE) & Evening(16-24) & WD $+\frac{1}{2} \mathrm{WE}$ & Day $+\frac{1}{2}$ Evening \\
Wednesday & & Night(0-8) & $\frac{1}{3} \mathrm{WD}+\mathrm{WE}$ & $\frac{1}{3} \mathrm{Day}+$ Evening \\
Thursday & & $\mathrm{WD}+\frac{1}{3} \mathrm{WE}$ & Day $+\frac{1}{3}$ Evening \\
Friday & & & \\
Saturday & & & \\
Sunday & & & \\
\hline
\end{tabular}

the network are defined in three ways: incoming, representing all phone calls made to a customer; outgoing, the phone calls made by a customer; and undirectional, when the previous distinction is not made. The weights of the edges are defined in four ways. These are the aggregated length of all phone calls, the total number of all phone calls, the normalized average of these two, and finally an indicator of whether a phone call was made or not. We refer to these as length, count, average and binary. Section 3.1 explicates how weights can be weighted in time with decay using equation 1 to confer more importance to more recent phone calls. This technique is also included in our network construction together with aggregating the weights normally. We call these two levels 'with decay' and 'simple weights'. For each network the combination of weights, directions and decay leads to 24 different combinations. The CDR data hold information about the date and time of each phone call. We use this information to segment the networks, thus building separate networks for phone calls made on each day of the week, during working days (WD) and weekends (WE) and during the day, evening and night. Finally, we combine times of the day and parts of the week in various ways to build more networks. Table 5 shows a summary of all the networks that were built in this way.

On a social level, a relationship between two people only exists when both call the other. As a result, some studies (Dasgupta et al., 2008; Haenlein, 2013) remove connections that were not reciprocal before applying SNA. To include this possibility in our exploration, we repeat the whole setup with non-reciprocal calls removed.

In total over 500 networks were built for the one dataset, which gives an indication of the scalability problem associated with these experiments and why more datasets and more relational learners are not included. The proxy classifier is subsequently applied to all the networks, and the performance of the resulting models compared. 
Table 6: Results of whole network measured in AUC and (lift at 0.5\%)

\begin{tabular}{|c|c|c|c|c|c|c|c|c|c|}
\hline & Length & Count & Average & Binary & Length & Count & Average & Binary & \\
\hline Whole & $0.748(3.21)$ & $0.748(3.21)$ & $0.504(1.16)$ & $0.748(3.21)$ & $0.755(3.21)$ & $0.748(3.21)$ & $0.743(3.05)$ & $0.748(3.21)$ & \multirow[b]{3}{*}{ 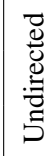 } \\
\hline Time-of-Day comb & $0.748(3.19)$ & $0.749(3.19)$ & $0.744(2.87)$ & $0.777(3.19)$ & $0.765(3.19)$ & $0.748(3.19)$ & $0.713(3.20)$ & $0.777(3.19)$ & \\
\hline Part-of-Week comb & $0.557(1.30)$ & $0.557(1.30)$ & $0.519(1.12)$ & $0.557(1.30)$ & $0.748(3.21)$ & $0.748(3.21)$ & $0.698(2.05)$ & $0.748(3.21)$ & \\
\hline Whole & $0.741(2.54)$ & $0.741(2.54)$ & $0.502(1.14)$ & $0.741(2.54)$ & $0.741(2.54)$ & $0.741(2.54)$ & $0.721(2.36)$ & $0.741(2.54)$ & \\
\hline Time-of-Day comb & $0.740(2.56)$ & $0.740(2.56)$ & $0.688(1.93)$ & $0.740(2.56)$ & $0.743(2.56)$ & $0.741(2.56)$ & $0.643(1.69)$ & $0.740(2.56)$ & \\
\hline Part-of-Week comb & $0.535(1.26)$ & $0.535(1.26)$ & $0.510(1.12)$ & $0.535(1.26)$ & $0.741(2.54)$ & $0.741(2.54)$ & $0.623(1.51)$ & $0.741(2.54)$ & \\
\hline Whole & $0.717(2.28)$ & $0.717(2.26)$ & $0.703(2.22)$ & $0.717(2.28)$ & $0.717(2.28)$ & $0.717(2.26)$ & $0.704(2.22)$ & $0.717(2.28)$ & \multirow{3}{*}{ 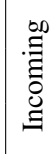 } \\
\hline Time-of-Day comb & $0.728(2.26)$ & $0.716(2.26)$ & $0.680(1.87)$ & $0.716(2.26)$ & $0.721(2.26)$ & $0.718(2.26)$ & $0.679(1.87)$ & $0.716(2.26)$ & \\
\hline Part-of-Week comb & $0.729(2.28)$ & $0.717(2.26)$ & $0.666(1.83)$ & $0.717(2.28)$ & $0.723(2.28)$ & $0.717(2.26)$ & $0.666(1.83)$ & $0.717(2.28)$ & \\
\hline & \multicolumn{4}{|c|}{ Simple weights } & \multicolumn{4}{|c|}{ With decay } & \\
\hline
\end{tabular}

\subsection{Results of Network Construction}

We first discuss the results of the whole network where non-reciprocal edges have not been removed.

Table 6 shows the performance of the relational learner when predicting churn measured in AUC and lift at $0.5 \%$.

For comprehensibility, we have chosen to show only three segmentations of the networks, namely the whole network, the best combination of part-of-week and the best combination of time-of-day. The best combination of part-of-week is the fourth one in Table 5 or $W D+\frac{1}{3} W E$ and the best combination of time-of-day is the first one in Table 5 which is $\frac{1}{2}$ Day + Evening. From Table 6 it is clear that the performance on the part-of-week combinations is lower than that on the other networks. In addition, performance on the whole network tends to be slightly better than on the time-of-day combination network. This result is the first interesting behavior that we can now extract. In some cases there is an increase in predictive capability by segmenting the network by part-of-week and also by time-of-day, with weekdays more important than weekends, and evenings more important than daytime. These results are consistent with the conclusion from our previous section: calling the close circle of the user (family and close friends) tends to be the most relevant factor when spreading churn influence. There is a greater chance that these calls occur in the evenings (after work) or during the weekdays, as, during the weekend, it is more likely that the users will be in the company of their close circle.

Given that such a weighting scheme can be used to improve predictive capability, and that this is closely related to the nature of the problem, then a theoretical approach of first propagating the 
network influence over the whole network (the second best approach), later analyzing extracting on whom the influence is exerted, and finally weighting the networks to reflect this group might bring an improvement in predictive capabilities of a few percentage points.

When comparing the definition of the edges - that is, the difference between performance on undirected, outgoing, and incoming networks - it is clear the undirected outperforms the other two. However, it is not as clear whether the outgoing or the incoming network is better. This result contradicts the findings of Haenlein (2013), where the outgoing edges are shown to have higher correlation with churn. Given that our study uses a far larger number of mobile phone users (representing all calls in a given time frame) instead of a selected sample - Haenlein (2013) uses 3431 users - we can venture that the directionality effect might occur only on some selected cliques, but in general it is only the presence of connectivity that is the main factor for allowing the spread of influence.

In addition, there seems to be a correlation between the edge definition and segmentation of the network, in that the performance of part-of-week network is higher for the incoming network and lower for the outgoing network, while the opposite holds for the time-of-day network.

Regarding the weights of the edges, the performance on the network with binary weights is almost consistently better or at least as good as on the networks with length and count, which have very similar performance. This indicates that it does not matter whether length or counts are used for the weights and that the simplest variant, binary weights, will result in models that are just as efficient. On the average network, performance is always the worst. This behavior hints at the importance of the existence of connectivity rather than the intensity of the communication between two users. As mentioned several times, it is those in the close circle of the user who are more at risk of churn when the user churns, so the number, and particularly the length of the call would be a poor proxy of the intensity of the relationship (a couple that lives together would probably not make very long calls to each other consistently, for example) and, as such, binary weights that reflect whether there is a connection or not would be sufficient to represent this relationship.

Finally, we compare performance on networks with and without decay on the weights. The networks with decay often slightly outperform the variant without decay, at least when performance is measured in AUC. This effect is not as clear in the table for lift at $0.5 \%$. This result, now consistent with Haenlein (2013), indicates that the influence of a churner is spread over the users that are in recent contact with the churner. This is quite obvious: the close circle is likely to be in permanent (so also recent) contact with the user, and users not in recent contact with the user would not have that 
Table 7: Results of reciprocal network measured in AUC and (lift at 0.5\%).

\begin{tabular}{|c|c|c|c|c|c|c|c|c|c|}
\hline & Length & Count & Average & Binary & Length & Count & Average & Binary & \\
\hline Whole & $0.681(1.83)$ & $0.681(1.83)$ & $0.676(1.81)$ & $0.681(1.83)$ & $0.681(1.83)$ & $0.681(1.83)$ & $0.676(1.81)$ & $0.681(1.83)$ & \multirow{3}{*}{ 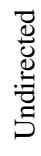 } \\
\hline Time-of-Day comb & $0.681(1.85)$ & $0.681(1.85)$ & $0.661(1.71)$ & $0.681(1.85)$ & $0.681(1.85)$ & $0.681(1.85)$ & $0.661(1.71)$ & $0.681(1.85)$ & \\
\hline Part-of-Week comb & $0.681(1.83)$ & $0.681(1.83)$ & $0.651(1.67)$ & $0.681(1.83)$ & $0.682(1.83)$ & $0.681(1.83)$ & $0.651(1.67)$ & $0.681(1.83)$ & \\
\hline Whole & $0.681(1.83)$ & $0.681(1.83)$ & $0.664(1.73)$ & $0.681(1.83)$ & $0.683(1.83)$ & $0.681(1.83)$ & $0.664(1.73)$ & $0.681(1.83)$ & \multirow[t]{3}{*}{ 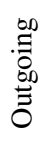 } \\
\hline Time-of-Day comb & $0.681(1.85)$ & $0.680(1.85)$ & $0.622(1.53)$ & $0.682(1.85)$ & $0.680(1.85)$ & $0.680(1.85)$ & $0.622(1.53)$ & $0.682(1.85)$ & \\
\hline Part-of-Week comb & $0.681(1.83)$ & $0.681(1.83)$ & $0.606(1.48)$ & $0.681(1.83)$ & $0.682(1.83)$ & $0.681(1.83)$ & $0.606(1.48)$ & $0.681(1.83)$ & \\
\hline Whole & $0.681(1.83)$ & $0.681(1.83)$ & $0.664(1.73)$ & $0.681(1.87)$ & $0.681(1.83)$ & $0.681(1.83)$ & $0.664(1.73)$ & $0.681(1.83)$ & \multirow{3}{*}{ 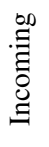 } \\
\hline Time-of-Day comb & $0.681(1.87)$ & $0.680(1.87)$ & $0.639(1.61)$ & $0.681(1.83)$ & $0.680(1.87)$ & $0.680(1.87)$ & $0.639(1.61)$ & $0.681(1.83)$ & \\
\hline Part-of-Week comb & $0.681(1.83)$ & $0.681(1.83)$ & $0.626(1.55)$ & $0.681(1.83)$ & $0.683(1.83)$ & $0.681(1.83)$ & $0.626(1.55)$ & $0.681(1.83)$ & \\
\hline & \multicolumn{4}{|c|}{ Simple weights } & \multicolumn{4}{|c|}{ With Decay } & \\
\hline
\end{tabular}

effect. Another interesting conclusion that can be extracted from this result is that the time frame for influencing is short, as our networks cover six months of calls at most.

We now turn to the results for networks with reciprocal edges only, which can be seen, measured in AUC and lift at $0.5 \%$, in Table 7. Overall, the difference in performance between the types of edges, types of segmentation, types of weights and with and without decay is much less evident than above, and in most cases there is no difference. It is possible to detect slightly better performance in the undirected network. However, the clear difference between these results and the results in Table 6 is that, by removing the non-reciprocal edges, the performance has decreased significantly.

To conclude, the use of the best performing network - that is, one which is constructed with binary weights, undirected and weighted in time - brings about a significant increase in performance, so the modeler has a large responsibility to correctly define the best network for the problem that is being tackled, as failure to do so will result in less predictive capability and therefore less potential gains.

\section{Scientific and Managerial Insights}

Applying SNA for CCP in telco is a complex process where multiple factors need to be taken into consideration. The results of the previous sections, however, can be used as guidelines on how to conduct this process in a practical way to achieve best performance. In addition to being relevant for business users, the results also have important implications for academics because experiments as extensive as these ones, for SNA-based CCP in telco, do not have precedence in the literature.

Firstly, the follow-up study on the effect of network construction on model performance suggests 
Table 8: Research Questions with Answers. Performance is measured using four measures: lift at $0.5 \%$ and $5 \%$, AUC, and EMP as described in subsection 3.4 .

\begin{tabular}{|c|c|c|c|c|}
\hline \multirow{4}{*}{ 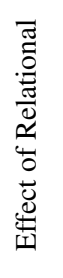 } & \multirow{4}{*}{ 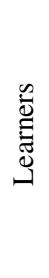 } & RQ1 & Which relational learners perform statistically different from the rest when predicting customer churn? & See Figure 5 \\
\hline & & RQ2 & Do some relational classifiers perform statistically better than the others? & Yes \\
\hline & & RQ3 & Is the performance of some collective inference methods statistically better than the others? & Yes \\
\hline & & RQ4 & $\begin{array}{l}\text { When predicting customer churn, do collective inference methods improve the predictive performance of rela- } \\
\text { tional classifiers? }\end{array}$ & No \\
\hline \multirow{2}{*}{ 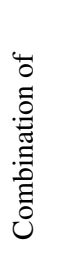 } & \multirow{2}{*}{ 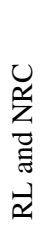 } & RQ5 & $\begin{array}{l}\text { Which non-relational classifier model performs best when predicting churn? A model built using network } \\
\text { features only, a model with relational learners scores only, or a model which is built with a combination of } \\
\text { both? }\end{array}$ & Both \\
\hline & & RQ6 & Which model type performs better when predicting churn? Relational learners or non-relational classifiers? & NRC \\
\hline
\end{tabular}

that how the network is defined does indeed make a difference to the performance of the models that

are applied to them. Networks with undirected edges and binary weights show the highest performance. In addition, more recent connections seem to matter more than older ones do. This is a very meaningful result for the day-to-day business user since it implies that simpler networks are just as good as more complex ones. Therefore, less effort needs to be made when filtering data and building networks, thus simplifying the process. Furthermore, as more recent connections are more important, less data is needed. Because of the lack of research on the effect of network construction on model performance, the consequences of our study are also important in an academic setting. They could furthermore help in understanding human behavior when it comes to social ties and churn.

Secondly, our extensive empirical comparison of the performance of relational learners and their combination with binary classifiers can be summarized with the six research questions and answers in Table 8. We compare all relational learners collectively, as well as their two components - relational classifiers and collective inference methods. Our tests reveal a small group of learners that perform better overall, and which are significantly better at predicting churn than the rest. The classifier, network-only link-based classifier proposed by Lu \& Getoor (2003) is the only one that significantly differs from the others, and it also proved to be the best performing one. This classifier uses the whole network to build a logistic regression model with link-based features from the network. Not only does it take into account information from a node's neighborhood when inferring a label, like the other classifiers, but also considers the joint information from the whole network. In fact, it is very similar to the logistic NRC model. Two of the collective inference methods show a significant 
difference from the rest. On the one hand, iterative classification is significantly worse than the other. depend on the purpose of the model and take into account how it is to be used. In CCP, this means accurately identifying the customers with the highest propensity to churn, since the loss of these customers will result in the highest loss for the provider. The commonly used AUC and lift measures do 
not take into account the profit and costs which inevitably accompany a retention campaign, in contrast to the Maximum Profit Measure (Verbeke et al., 2012, Verbraken et al., 2013). This profit-centric measure evaluates models by optimizing the profit of the model and the fraction of the customers that should be offered promotions in order to get the highest profit.

\section{Conclusion}

\subsection{Main Contributions}

Social network analysis has been shown to make a difference when applied to customer churn prediction in telco. From the definition of the network itself, to extracting insightful features, and building and evaluating the predictive models, multiple decisions need to be made at every step of the process. In this study, we performed tests on some of these possibilities, investigated the impact of the construction of networks and the applied techniques on model performance and offered guidelines on how this can be done optimally. Overall our study offers two main contributions to the existing literature.

Our first main contribution is an empirical comparison of the performance of relational learners and their combination with binary classifiers when predicting customer churn in telco.The results were evaluated on a large number of CDR datasets, which allowed us to apply statistical tests to assess the significance of our results. The CDR datasets originated from a number of telcos across the world and varied in both size and churn rate. The performance of the resulting models was evaluated with four measures, which gave consistent and robust results. A benchmarking of SNA methods for churn prediction in telco on this scale does not have precedence in the literature to the best of our knowledge. An overview of our findings can be seen in Table 8 .

The second main contribution of this paper is an exploratory study of various network architectures and how they effect model performance. As far as we know, this has not been studied explicitly in the context of customer churn before. Our results imply that network definition does matter for performance, with undirected, binary network resulting in the highest performance and recent connections having greater importance than older ones do. Furthermore, the inclusion of contextual information that leads to an appropriate definition of the network is key in assuring maximum predictive capabilities. 


\subsection{Future Work}

Although we have been able to provide answers to the research questions, many more still remain

775

\footnotetext{
Athanassopoulos, A. D. (2000). Cust
business research, 47, 191-207.
} roaming information would have. to achieve best performance.

\section{References} unanswered and can be viewed as objectives for future studies.

First of all, a vital component of each dataset was missing in our analyses: local variables. These represent information about the customers as isolated entities without relations to other customers, such as demographic information, pricing plans, and financials, among others. Previous studies have shown that models built with these variables alone perform worse than when network features are included as well (Verbeke et al., 2014). We would like to see how this effect presents itself together with relational learners and include it in the experimental design of our study.

Secondly, only information about phone calls was used to build networks throughout the study. It would be interesting to see what effect including networks created from text messages and even

Thirdly, we have provided an insight into how network construction might effect model performance. This was only done by the means of one relational learner and one dataset. A more comprehensive study with more datasets and other types of models would offer the possibility to evaluate this effect statistically and, as a result, provide guidelines on how to construct the network to achieve the highest performance. In addition, since we only used one classifier, we were unable to verify whether there is correlation between network construction and classifier when it comes to model performance. Finally, an in-depth study would allow us to determine the optimal way of constructing networks, with regard to edges and weights, and to determine the actual timespan of CDR data that are needed

Ahn, J.-H., Han, S.-P., \& Lee, Y.-S. (2006). Customer churn analysis: Churn determinants and mediation effects of partial defection in the korean mobile telecommunications service industry. Telecommunications policy, 30, 552-568.

Ali, Ö. G., \& Arttürk, U. (2014). Dynamic churn prediction framework with more effective use of rare event data: The case of private banking. Expert Systems with Applications, 41, 7889-7903.

Anil Kumar, D., \& Ravi, V. (2008). Predicting credit card customer churn in banks using data mining. International Journal of Data Analysis Techniques and Strategies, 1, 4-28. 
Backiel, A., Baesens, B., \& Claeskens, G. (2014). Mining telecommunication networks to enhance customer lifetime predictions. In Artificial Intelligence and Soft Computing (pp. 15-26). Springer.

Backiel, A., Verbinnen, Y., Baesens, B., \& Claeskens, G. (2015). Combining local and social network classifiers to improve churn prediction. In Proceedings of the 2015 IEEE/ACM International Conference on Advances in Social Networks Analysis and Mining 2015 (pp. 651-658). ACM.

Baesens, B., Van Vlasselaer, V., \& Verbeke, W. (2015). Fraud Analytics Using Descriptive, Predictive, and Social Network Techniques: A Guide to Data Science for Fraud Detection. Wiley and SAS Business Series. Wiley.

Baras, D., Ronen, A., \& Yom-Tov, E. (2014). The effect of social affinity and predictive horizon on churn prediction using diffusion modeling. Social Network Analysis and Mining, 4, 1-12.

Benedek, G., Lublóy, Á., \& Vastag, G. (2014). The importance of social embeddedness: Churn models at mobile providers. Decision Sciences, 45, 175-201.

Berson, A., \& Smith, S. J. (2002). Building data mining applications for CRM. McGraw-Hill, Inc.

Chen, Z.-Y., Fan, Z.-P., \& Sun, M. (2012). A hierarchical multiple kernel support vector machine for customer churn prediction using longitudinal behavioral data. European Journal of operational research, 223, 461-472.

Coussement, K., \& Van den Poel, D. (2008). Churn prediction in subscription services: An application of support vector machines while comparing two parameter-selection techniques. Expert systems with applications, 34, 313-327.

Dasgupta, K., Singh, R., Viswanathan, B., Chakraborty, D., Mukherjea, S., Nanavati, A. A., \& Joshi, A. (2008). Social ties and their relevance to churn in mobile telecom networks. In Proceedings of the 11th international conference on Extending database technology: Advances in database technology (pp. 668-677). ACM.

De Bock, K. W., \& Van den Poel, D. (2011). An empirical evaluation of rotation-based ensemble classifiers for customer churn prediction. Expert Systems with Applications, 38, 12293-12301.

Demsar, J. (2006). Statistical comparisons of classifiers over multiple data sets. The Journal of Machine Learning Research, 7, 1-30.

Dierkes, T., Bichler, M., \& Krishnan, R. (2011). Estimating the effect of word of mouth on churn and cross-buying in the mobile phone market with markov logic networks. Decision Support Systems, 51, 361-371.

Fawcett, T. (2006). An introduction to roc analysis. Pattern recognition letters, 27, 861-874.

Ganesh, J., Arnold, M. J., \& Reynolds, K. E. (2000). Understanding the customer base of service providers: an examination of the differences between switchers and stayers. Journal of marketing, 64, 65-87.

Glady, N., Baesens, B., \& Croux, C. (2009). Modeling churn using customer lifetime value. European Journal of Operational Research, 197, 402-411.

Guillén, M., Nielsen, J. P., Scheike, T. H., \& Pérez-Marín, A. M. (2012). Time-varying effects in the analysis of customer loyalty: A case study in insurance. Expert Systems with Applications, 39, 3551-3558.

Günther, C.-C., Tvete, I. F., Aas, K., Sandnes, G. I., \& Borgan, Ø. (2014). Modelling and predicting customer churn from an insurance company. Scandinavian Actuarial Journal, 2014, 58-71. 
Haenlein, M. (2013). Social interactions in customer churn decisions: The impact of relationship directionality. International Journal of Research in Marketing, 30, 236-248.

Hand, D. J. (2009). Measuring classifier performance: a coherent alternative to the area under the roc curve. Machine learning, 77 , $103-123$.

Huang, B., Kechadi, M. T., \& Buckley, B. (2012). Customer churn prediction in telecommunications. Expert Systems with Applications, $39,1414-1425$

Hung, S.-Y., Yen, D. C., \& Wang, H.-Y. (2006). Applying data mining to telecom churn management. Expert Systems with Applications, $31,515-524$.

Jensen, D., Neville, J., \& Gallagher, B. (2004). Why collective inference improves relational classification. In Proceedings of the tenth ACM SIGKDD international conference on Knowledge discovery and data mining (pp. 593-598). ACM.

Khan, A. A., Jamwal, S., \& Sepehri, M. (2010). Applying data mining to customer churn prediction in an internet service provider. International Journal of Computer Applications, 9, 8-14.

Kim, K., Jun, C.-H., \& Lee, J. (2014). Improved churn prediction in telecommunication industry by analyzing a large network. Expert Systems with Applications, 41, 6575-6584.

Kusuma, P. D., Radosavljevik, D., Takes, F. W., \& van der Putten, P. (2013). Combining customer attribute and social network mining for prepaid mobile churn prediction. In Proc. the 23rd Annual Belgian Dutch Conference on Machine Learning (BENELEARN) (pp. $50-58)$.

Larivière, B., \& Van den Poel, D. (2004). Investigating the role of product features in preventing customer churn, by using survival analysis and choice modeling: The case of financial services. Expert Systems with Applications, 27, 277-285.

Lessmann, S., Baesens, B., Seow, H.-V., \& Thomas, L. C. (2015). Benchmarking state-of-the-art classification algorithms for credit scoring: An update of research. European Journal of Operational Research, 247, 124-136.

Lu, Q., \& Getoor, L. (2003). Link-based classification. In ICML (pp. 496-503). volume 3.

Macskassy, S. A., \& Provost, F. (2007). Classification in networked data: A toolkit and a univariate case study. The Journal of Machine Learning Research, 8, 935-983.

Miritello, G., Moro, E., Lara, R., Martínez-López, R., Belchamber, J., Roberts, S. G., \& Dunbar, R. I. (2013). Time as a limited resource: Communication strategy in mobile phone networks. Social Networks, 35, 89-95.

Modani, N., Dey, K., Gupta, R., \& Godbole, S. (2013). Cdr analysis based telco churn prediction and customer behavior insights: A case study. In Web Information Systems Engineering-WISE 2013 (pp. 256-269). Springer.

Mozer, M. C., Wolniewicz, R., Grimes, D. B., Johnson, E., \& Kaushansky, H. (2000). Predicting subscriber dissatisfaction and improving retention in the wireless telecommunications industry. Neural Networks, IEEE Transactions on, 11, 690-696.

Naboulsi, D., Fiore, M., Ribot, S., \& Stanica, R. (2015). Large-scale mobile traffic analysis: a survey. IEEE Communications Surveys \& Tutorials, 18, 124-161.

Nanavati, A. A., Singh, R., Chakraborty, D., Dasgupta, K., Mukherjea, S., Das, G., Gurumurthy, S., \& Joshi, A. (2008). Analyzing the structure and evolution of massive telecom graphs. IEEE Transactions on Knowledge and Data Engineering, 20, 703-718. 
Neslin, S. A., Gupta, S., Kamakura, W., Lu, J., \& Mason, C. H. (2006). Defection detection: Measuring and understanding the predictive accuracy of customer churn models. Journal of marketing research, 43, 204-211.

Newman, M. (2010). Networks: an introduction. OUP Oxford.

Ngonmang, B., Viennet, E., \& Tchuente, M. (2012). Churn prediction in a real online social network using local community analysis. In Proceedings of the 2012 International Conference on Advances in Social Networks Analysis and Mining (ASONAM 2012) (pp. 282-288). IEEE Computer Society.

Park, S.-H., Huh, S.-Y., Oh, W., \& Han, S. P. (2012). A social network-based inference model for validating customer profile data. MIS Quarterly, 36, 1217-1237.

Phadke, C., Uzunalioglu, H., Mendiratta, V. B., Kushnir, D., \& Doran, D. (2013). Prediction of subscriber churn using social network analysis. Bell Labs Technical Journal, 17, 63-75.

Van den Poel, D., \& Lariviere, B. (2004). Customer attrition analysis for financial services using proportional hazard models. European journal of operational research, 157, 196-217.

Raeder, T., Lizardo, O., Hachen, D., \& Chawla, N. V. (2011). Predictors of short-term decay of cell phone contacts in a large scale communication network. Social Networks, 33, 245-257.

Rehman, A., \& Raza Ali, A. (2015). Customer churn prediction, segmentation and fraud detection in telecommunication industry, .

Richter, Y., Yom-Tov, E., \& Slonim, N. (2010). Predicting customer churn in mobile networks through analysis of social groups. In SDM (pp. 732-741). volume 2010.

Sarraute, C., Brea, J., Burroni, J., \& Blanc, P. (2015). Inference of demographic attributes based on mobile phone usage patterns and social network topology. Social Network Analysis and Mining, 5, 1-18.

Sen, P., Namata, G., Bilgic, M., Getoor, L., Galligher, B., \& Eliassi-Rad, T. (2008). Collective classification in network data. AI magazine, 865 $29,93$.

Tomar, V., Asnani, H., Karandikar, A., Chander, V., Agrawal, S., \& Kapadia, P. (2010). Social network analysis of the short message service. In 2010 National Conference on Communications, Chennai (pp. 1-5). Citeseer.

Tufféry, S. (2011). Data mining and statistics for decision making. John Wiley \& Sons.

Vafeiadis, T., Diamantaras, K. I., Sarigiannidis, G., \& Chatzisavvas, K. C. (2015). A comparison of machine learning techniques for customer churn prediction. Simulation Modelling Practice and Theory, 55, 1-9.

Van Vlasselaer, V., Bravo, C., Caelen, O., Eliassi-Rad, T., Akoglu, L., Snoeck, M., \& Baesens, B. (2015). Apate: A novel approach for automated credit card transaction fraud detection using network-based extensions. Decision Support Systems, 75, 38-48.

Van Vlasselaer, V., Eliassi-Rad, T., Akoglu, L., Snoeck, M., \& Baesens, B. (2016). Gotcha! network-based fraud detection for social security fraud. Management Science, accepted, .

Verbeke, W., Dejaeger, K., Martens, D., Hur, J., \& Baesens, B. (2012). New insights into churn prediction in the telecommunication sector: A profit driven data mining approach. European Journal of Operational Research, 218, 211-229.

Verbeke, W., Martens, D., \& Baesens, B. (2014). Social network analysis for customer churn prediction. Applied Soft Computing, 14, 431-446. 
Verbraken, T., Bravo, C., Weber, R., \& Baesens, B. (2014). Development and application of consumer credit scoring models using profitbased classification measures. European Journal of Operational Research, 238, 505-513.

Verbraken, T., Verbeke, W., \& Baesens, B. (2013). A novel profit maximizing metric for measuring classification performance of customer churn prediction models. Knowledge and Data Engineering, IEEE Transactions on, 25, 961-973.

Xie, Y., Li, X., Ngai, E., \& Ying, W. (2009). Customer churn prediction using improved balanced random forests. Expert Systems with Applications, 36, 5445-5449.

Zhang, X., Zhu, J., Xu, S., \& Wan, Y. (2012). Predicting customer churn through interpersonal influence. Knowledge-Based Systems, 28, 97-104.

Zhu, T., Wang, B., Wu, B., \& Zhu, C. (2011). Role defining using behavior-based clustering in telecommunication network. Expert Systems with Applications, 38, 3902-3908.

\section{Appendix A Abbreviations}

A relational learner is constructed by combining a relational classifier with a collective inference method. This is denoted by writing CI-RC. For example, gibbs-nlb means the combination of Gibbs sampling with the network only link based classifier. 
Table 9: Table of Abbreviations

\begin{tabular}{|c|c|c|}
\hline & Abbreviation & Meaning \\
\hline \multirow{8}{*}{ General } & SNA & Social Network Analysis \\
\hline & $\mathrm{CCP}$ & Customer Churn Prediction \\
\hline & CDR & Call-Detail Records \\
\hline & RL & Relational Learner \\
\hline & $\mathrm{RC}$ & Relational Classifier \\
\hline & $\mathrm{CI}$ & Collective Inference Method \\
\hline & NRC & Non Relational Classifier \\
\hline & RFM & Recency, Frequency and Monetary \\
\hline \multirow{4}{*}{$\mathrm{RC}$} & WVRN/wvrn & Weighted Vote Relational Neighbor Classifier \\
\hline & $\mathrm{CDRN} / \mathrm{cdrn}$ & Class Distribution Relational Neighbor Classifier \\
\hline & $\mathrm{NLB} / \mathrm{nlb}$ & Network Only Link Based Classifier \\
\hline & SPA RC/spaRC/sp & Spreading Activation Relational Classifier \\
\hline \multirow{6}{*}{$\mathrm{CI}$} & gib/gibbs & Gibbs Sampling \\
\hline & $\mathrm{IC} / \mathrm{ic}$ & Iterative Classification \\
\hline & $\mathrm{RL} / \mathrm{rl}$ & Relaxation Labelling \\
\hline & RLSA/rlsa & Relaxation Labelling with Simulated Annealing \\
\hline & SPA CI/spaCI/sp & Spreading Activation Collective Inference Method \\
\hline & NO & No/NO Collective Inference Method \\
\hline \multirow{3}{*}{ NRC } & $\log$ & Logistic Regression \\
\hline & $\mathrm{RF}$ & Random Forest \\
\hline & NN & Nerual Networks \\
\hline \multirow{2}{*}{ Measure } & MP & Maximum Profit \\
\hline & EMP & Expected Maximum Profit \\
\hline
\end{tabular}

